\title{
Integrative network analysis of rifampin- regulated miRNAs and their functions in human hepatocytes
}

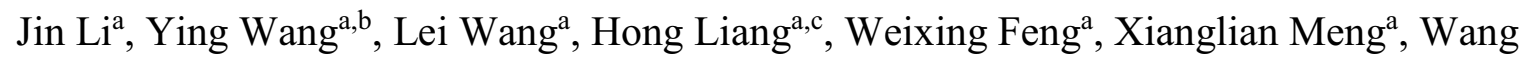 \\ Cong ${ }^{\mathrm{a},{ }^{*}}$ and Yunlong Liu ${ }^{\mathrm{a},{ }^{*}}$ \\ ${ }^{a}$ College of Automation, Harbin Engineering University, 145 Nantong Street, Nangang District, \\ Harbin, Heilongjiang, 150001, China \\ ${ }^{b}$ Network Information Center, Qiqihar University, No. 42, Wenhua Street, Qiqihar, Heilongjiang, \\ 161006, China \\ ${ }^{c}$ Department of Medical and Molecular Genetics, Indiana University School of Medicine, 355 West \\ 16th Street, Suite 4100, Indianapolis, IN 46202, USA
}

\begin{abstract}
Rifampin is an important drug used in the treatment of tuberculosis, and it increases the drug metabolism in human hepatocytes. Previous studies have shown that rifampin can indirectly influence drug deposition through the regulation of molecular interactions of miRNA, PXR and other genes. The potential functions of miRNAs associated with rifampininduced drug disposition are poorly understood. In this study, significantly differentially expressed miRNAs (SDEM) were extracted and used to predict the miRNA-regulated co-expression target genes (MCeTG). Additionally, a miRNA-regulated co-expressed protein interaction network (MCePIN) was constructed for SDEM by extending from the protein interaction network (PIN). The functioning of the miRNAs were analyzed using GO analysis and KEGG pathway enrichment analysis. A total of 20 miRNAs belonging to SDEM were identified, and 632 miRNA-regulated genes were predicted. The MCePIN was constructed by extending from PIN, and 10 miRNAs and 33 genes that are relevant to 7 functions, including response to wounding, wound healing, response to drug, defense response, inflammatory response, liver development and drug metabolism, were discerned. The results provided by this study offer valuable insights into the effect of rifampin on miRNAs, genes and protein levels.
\end{abstract}

Keywords: Rifampin, miRNA, gene, hepatocyte, PPI network, P450

\section{Introduction}

Rifampin is an effective antimicrobial drug that is usually used to treat tuberculosis and other infections [1]. Rifampin works by inducing the drug-metabolizing enzymes and transporters [2]. Rifampin, a typical ligand of the Pregnane X receptor (PXR), has been shown to strongly stimulate

\footnotetext{
* Address for correspondence: Wang Cong, Harbin Engineering University, 145, Nantong Street, Nangang District, Harbin, Heilongjiang, 150001, China. Tel.: 86-451-82519998; Fax: 86-451-82519253; E-mail: cwcy9156@163.com.

Yunlong Liu, Harbin Engineering University, 145, Nantong Street, Nangang District, Harbin, Heilongjiang, 150001, China. Tel.: 86-451-82569412; Fax: 86-451-82589400; E-mail:yunliu@iupui.edu.
} 
drugs by activating the PXR [3-5], and is also an inducer of many cytochrome P450 (p450) enzymes [2]. And RXR regulator mediates the gene expression including some genes of P450 [6].

MicroRNAs (miRNAs) are a class of $22 \mathrm{nt}$ non-coding RNAs that typically regulate protein-coding genes at the post-transcription level [7,8], and are predicted to regulate more than one-third of the protein-genes in the human genome [9]. Rifampin-induced miRNA expression changes are correlated with rifampin treatment changes in PXR and P450 [6, 10-12]. MiRNA can indirectly influence rifampin-induced drug deposition through gene regulation [11, 13-15]. Proteins are synthesized from mRNA templates through a highly conserved process, many studies have reported that the functions of miRNAs can be understood by analyzing miRNAs-regulated mRNA-related PIN [16-18].

In this study, miRNA and mRNA expression profiles were combined to better understand the functions of rifampin-regulated miRNAs and their potential molecular mechanisms within human hepatocytes. Following this initial analysis, the target genes of each differentially expressed miRNA were predicted. Furthermore, MCeTG were extracted, and rifampin-induced miRNAs-regulated coexpression PIN was constructed by integrating the PPI network. In addition, the functioning of the different miRNAs was analyzed by assessing the underlying MCePIN of each miRNA using functional enrichment analysis. The results provided by this study offer new insights into the miRNA and miRNA function when interacting with rifampin.

\section{Materials and methods}

\subsection{Data source}

The mRNA expression dataset $(n=12780)$ and miRNA expression dataset $(n=334)$ were obtained as in Ramanoorthy, et al [9]. Briefly, seven hepatocyte samples from seven different human were treated with rifampin, and the RNA was isolated from tye samples using the miRNease kit. The expressions of 754 miRNAs were measured using the Taqman OpenArray Human miRNA Panel. The mRNA expressions were obtained using EZBead preparation, Next-Gene sequencing, read quality assessment, sequence alignment and RNA-Seq differential expression analysis.

\subsection{Statistical analysis}

Benjamini-Hochberg correction was applied to control for the false discovery rate (FDR) [19]. A pvalue was calculated for each miRNA and each gene of two groups (normal and rifampin-treated samples) with an unpaired, two-tailed Student's t test.

\subsection{Target gene prediction}

The miRNAs were significantly different when the $\mathrm{p}$-value $<0.01$. The predicted miRNA target genes were predicted in Targetscan [20, 21], miRanda [22] and PicTar [23] using RmiR.hsa, which retrieved the targets or the miRNAs in miRNA target databases for given miRNAs or genes without any threshold. BioMart [24] was used to convert gene Entrez ID into the gene Symbol.

\subsection{Constrction of MCePIN}

The Human Protein Reference Database (HPRD) [25] includes 30047 proteins and 41327 protein- 
protein interactions. The human liver protein interaction network (HLPN) is composed of 3484 interactions among 2582 proteins relevant to human liver. The MCePIN was created through the integration from the proteins and interactions of the HPRD and HLPN. However, duplicates were excluded [26]. The cytoscape [27], version 3.0.2 software was used to generate the network.

\subsection{Enrichment analysis of modules}

Enrichment analysis of the biological processes and pathways of the MCePIN was processed with the Database for Annotation, Visualization and Integrated Discovery (DAVID), which is a tool for biological interpretation of genes. Functional classification of important gene pathways was carried out using the Gene Ontology (GO) and Kyoto Encyclopedia of Genes and Genomes (KEGG).

\section{Results and discussions}

\subsection{MCePIN}

MCePIN was constructed using the significantly different expressed miRNAs, their co-expressed significantly different expressed target genes and the interactions between them.

The differentially expressed miRNAs were retrieved based on a $\mathrm{p}$-value $(\mathrm{p}<0.05)$. The upregutalted and downregulated miRNAs were identified using threshold (Fold Change $>1$ ) and threshold (Fold Change $<1$ ), respectively. There were 20 miRNAs, including 12 upregulated miRNAs and 8 downregulated miRNAs left (Table 1).

To extract target genes of 20 miRNAs in a liberal standard, Pictar, Targetscan and miRanda databases were used without setting any threshold. The target genes of the 20 miRNAs were the mixed dataset of the results from the three databases. In this analysis, our primary focus was on the genes in the gene expression profile. Therefore, 6211 genes were extracted, and the negatively regulated target genes of each miRNA were obtained using the fold change that represents a measure of expression changes. As a result, 4115 co-expression genes of the 20 miRNAs were retained. From this, there were 632 significantly differentially expressed genes, and 1187 co-expression miRNA-gene pairs were identified. The HPRD and HLPN were integrated by merging all the proteins and interactions, and then removing the duplicates. The human PIN included 42521 interactions among 10210 proteins. Table 1

Differentially expressed miRNA dataset

\begin{tabular}{llllll}
\hline miRNA & P-value & Fold Change & miRNA & p-value & Fold Change \\
\hline upregulated & & & upregulated & & \\
\hline miR-886-3p & 0.00017781 & 1.56454118 & miR-660 & 0.02971142 & 1.26423062 \\
\hline miR-766 & 0.00746736 & 1.42202073 & miR-638 & 0.0302057 & 1.67681216 \\
\hline miR-92a & 0.01687698 & 1.13186208 & miR-25 & 0.0337675 & 1.27581638 \\
\hline miR-107 & 0.01770697 & 2.18865184 & miR-616 & 0.04464574 & 1.33365615 \\
\hline miR-30d\# & 0.01945112 & 1.15621932 & miR-576-3p & 0.04528093 & 2.19157158 \\
\hline miR-335 & 0.02415006 & 1.33006108 & miR-218 & 0.04993545 & 1.9012223 \\
\hline Downregulated & & & & & \\
\hline miR-186 & 0.00177975 & 0.83561977 & miR-320 & 0.03756323 & 0.77870999 \\
\hline miR-361 & 0.01111667 & 0.7086572 & miR-202 & 0.03960494 & 0.59880068 \\
\hline miR-95 & 0.02185477 & 0.63201986 & miR-200b\# & 0.04256355 & 0.60539667 \\
\hline miR-345 & 0.0239214 & 0.81506025 & let-7g & 0.04347655 & 0.84016356 \\
\hline
\end{tabular}


Based on the interactions in the human PIN, the relationships of the 632 proteins were established. Specifically, the miRNA-regulated PINs were extended by choosing the first level proteins nodes of genes. So, the MCePIN was constructed from 11219 interactions among 20 miRNAs and 632 proteins.

Among the 1187 co-expression miRNA-gene pairs, miR-660 negatively regulated the most genes (136), and miR-886 negatively regulated the least genes (12). In this analysis, the rifampin-associated genes were further to reveal potential miRNA-related PINs and the functions of miRNAs.

\subsection{Enrichment analysis of miRNA functions}

The biological classification tool DAVID, based on GO and KEGG, was used to describe the functional classification and signal pathways of the miRNAs and MCePIN genes. Based on the extension of network with PIN, GO terms and KEGG keys pathways associated with drug-induced were excluded. Functional annotation clustering was used to extract the concerned functions, and Go terms and KEGG pathways that contained "drug", "liver", "metabolic", "response", "wound" and "stimulus" were defined as drug-related functions. The results of the enriched biological processes for the genes and proteins related to the rifampin are listed in Table 2.

Table 2

Enrichment analysis of miRNA-regulated genes and proteins in MCePIN

\begin{tabular}{lllll}
\hline DAVID & description & Gene & protein & p-value \\
\hline GO:0009611, response to wounding & a stimulus indicating damage to the organism & 22 & 186 & $6.0 \mathrm{E}-26$ \\
\hline GO:0042060, wound healing & restore integrity to a damaged tissue & 7 & 85 & $7.7 \mathrm{E}-18$ \\
\hline GO:0042493, response to drug & a result of a drug stimulus & 15 & 77 & $1.2 \mathrm{E}-13$ \\
\hline GO:0006952, defense response & $\begin{array}{l}\text { response to the presence of a foreign body or } \\
\text { the occurrence of an injury }\end{array}$ & 19 & 143 & $4.6 \mathrm{E}-5$ \\
\hline GO:0006954, inflammatory response & $\begin{array}{l}\text { The immediate defensive reaction to infection } \\
\text { or injury }\end{array}$ & 16 & 96 & $8.6 \mathrm{E}-4$ \\
\hline $\begin{array}{l}\text { GO:0001889, liver development } \\
\text { progression of the liver over time }\end{array}$ & $\begin{array}{l}\text { Drug metabolism } \\
\begin{array}{l}\text { KEGG_PATHWAY, Drug } \\
\text { metabolism-P450 }\end{array}\end{array}$ & 6 & 21 & $5.4 \mathrm{E}-6$ \\
\hline
\end{tabular}

Table 3

The rifampin-associated miRNAs and genes of MCePIN

\begin{tabular}{|c|c|}
\hline function & miRNA \\
\hline response to wounding & $\begin{array}{lc}\text { miR-107, miR-186, miR-218, miR- AHSG, IL1RAP, IL1RN, THBS1, F8, IL10RB, } \\
\text { 576-3p, miR-886-3p, miR-335, miR- IL20RB, SGMS1, P2RX7, CD55, CXCL13, HIF1A, } \\
\text { 616, miR-766 } & \text { RELA, IGF1, PLSCR4, SLC1A2, TPM1 } \\
\end{array}$ \\
\hline wound healing & $\begin{array}{l}\text { Let-7g, miR-107, miR-218, miR-576- } \text { HBEGF, SYT7, PLSCR4, TPM1, IGF1, F8 } \\
\text { 3p, miR-766, miR-886-3p }\end{array}$ \\
\hline response to drug & $\begin{array}{l}\begin{array}{l}\text { miR-218, miR-766, miR-886-3p, miR- ABAT, CAV1, SLC1A2, BCHE, CAV2, HMGCS1, } \\
\text { 660, miR-576-3p } \\
\text { PLIN2, PPARG, P2RX7 }\end{array} \\
\end{array}$ \\
\hline defense response & $\begin{array}{llll}\text { miR-576-3p, miR-335, miR-186, miR- CD55, CXCL13, HIF1A, RELA, AHSG, IL1RAP, } & \text { IL1RN, THBS1, F8, IL10RB, IL20RB, SGMS1, } \\
886-3 p & \text { PPARG, P2RX7 } & & \end{array}$ \\
\hline inflammatory response & $\begin{array}{l}\text { CD55, CXCL13, HIF1A, RELA, AHSG, IL1RAP, } \\
\text { IL1RN, THBS1, F8, IL10RB, IL20RB, SGMS1 }\end{array}$ \\
\hline liver development & ONECUT2, ARF6, SP3, TGFBR3, CEBPA, RELA \\
\hline Drug metabolism-P450 & miR-107, miR-335, miR-186 \\
\hline
\end{tabular}


Response to wounding, wound healing, response to drug, defense response, inflammatory response, liver development and drug metabolism are the seven different functions that were identified Table 2). The rifampin-associated miRNAs and genes are listed in Table 3.

A total of 33 different rifampin-associated genes were identified (Table 3). Among them, P450 and CYP3A5 have been reported to have important roles in drug metabolism [28-30], and SP3, ARF6, CEBPA, RELA are responsible for liver development [31-34]. In addition, CXCL13 and RELA have been previously reported as inflammatory markers $[35,36]$.

The ten rifampin-associated miRNAs that targeted the 33 genes are listed as follow: miR-107, miR186, miR-218, miR-576-3p, miR-886-3p, miR-335, miR-616, miR-766, miR-218 and Let-7g. Previous studies have reported that rifampin induced expression change of genes and miRNAs $[9,10]$. These results suggest that miR-107, miR-186, miR-218, miR-576-3p, miR-886-3p, miR-335, miR-616 and miR-766 were correlated with response to wounding via direct or indirect mechanisms through regulating genes. Additionally, let-7g, miR-107, miR-218, miR-576-3p, miR-799 and miR-886-3p were related to wound healing via induced 6 genes. Further, miR-218, miR-766, miR-886-3p, miR660 and miR-576-3p enriched in response to drug, and miR-107 and miR-186 were candidates for participating in liver development. Similarly, miR-107, miR-335 and miR-186 were suggested to be related to drug metabolism through the regulation of ADH1B and CYP3A5 [37].

\subsection{Analysis of rifampin-associated miRNAs and negatively regulated genes}

To reveal the rifampin-associated miRNAs and genes, the miRNA-genes co-expression network was constructed (Figure 1).

There were 10 miRNAs that negatively regulated the 33 genes, and 62 miRNA-gene pairs in the network. To focus on the rifampin-associated miRNAs, miR-576-30, which controlled 11 genes, was designated as the most important in the network, and is suggested to participate in response to wounding, wound healing, response to drug, defense response and inflammatory response. The miR186 negatively regulated 7 genes, including CYP3A5, and it is suggested that miR-186 associated with the drug metabolic process. Although miR-335 did not negatively regulate CYP3A5, which is relevant

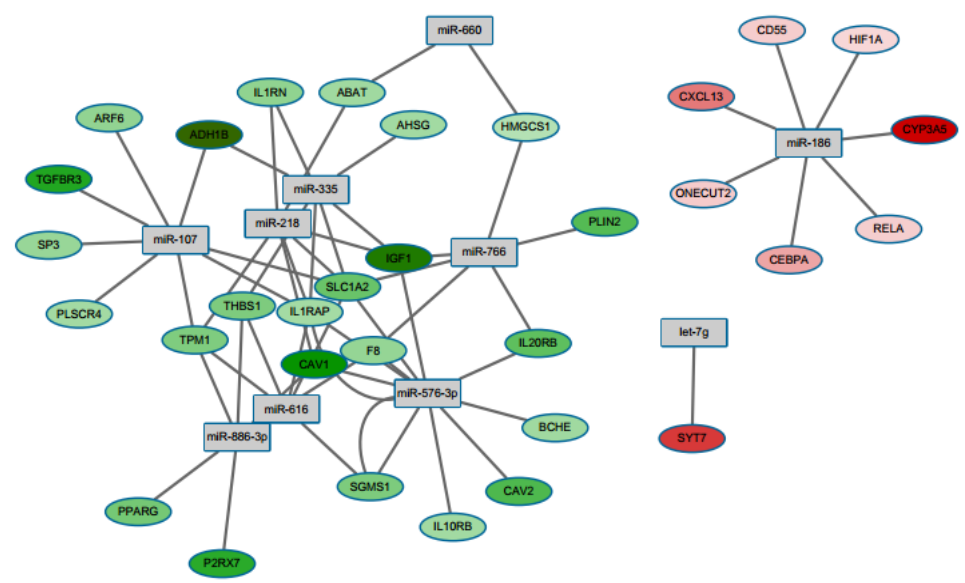

Fig. 1. Key miRNA-genes co-expression network. The yellow and grey rectangles represent the downregulated and upregulated miRNAs, respectively. The circles express the miRNA-regulated genes, and the regulatory relationships are denoted by colors, in which red and green indicate upregulated and downregulated genes, respectively. The depth of the color represents the size of the fold change. 
to drug metabolism, it did negatively control ADH1B, which is also related to drug metabolism.

The 33 genes performed the functions directly or indirectly through proteins. ADH1B, IGF1, CAV1, and SGMS1 had the major quantity changes, and were regulated by multiple miRNAs. CYP3A5 is an important gene that takes part in drug metabolism and differentially expressed. These results suggest that these five genes have important functions in the rifampin-induced biological process.

\section{Conclusion}

To understand the rifampin-induced differentially expressed miRNAs and their functions, the miRNA and gene profile were integrated to identify significant differential miRNA and miRNAs negatively regulated genes, further to constructed MCePIN by PIN. Consequently, seven functions were extracted through GO analysis and KEGG pathway enrichment. Moreover, it is suggested that miRNAs and genes are associated with each function. As a whole, the results suggest that rifampin contributes to changes in the expression of multiple miRNAs. It provides evidence for an important regulatory function of miRNAs in the rifampin-induced biological process in human hepatocytes.

\section{Acknowledgment}

The authors would like to acknowledge the financial support of the National Key Scientific Instrument and Equipment Development Projects of China (2012YQ04014001 and 2012YQ04014010), National Natural Science Foundation of China (61071174), Fundamental Research Funds for the Central Universities (HEUCFT1102, HEUCFT1302 and HEUCFX41303), Science and technology Research Program of Heilongjiang Educational Committee (12541898).

\section{References}

[1] C.R.C. Anne, M. Baciewicz, Christopher K. Finch and Timothy H. Self, Update on rifampin and rifabutin drug interactions, American Journal of the Medical Sciences 335 (2008), 126-136.

[2] M.D.J. James M. Rael, Marc E. Lippman and David A. Flockhart, Rifampin is a selective, pleiotropic inducer of drug metabolism, The Journal of Pharmacology and Experimental Therapeutics 299 (2001), 849-857.

[3] J. Chen and K. Raymond, Roles of rifampicin in drug-drug interactions: Underlying molecular mechanisms involving the nuclear pregnane X receptor, Annals of Clinical Microbiology and Antimicrobials 5 (2006), 3-13.

[4] S.A. Kliewer, B. Goodwin and T.M. Willson, The nuclear pregnane X receptor: A key regulator of xenobiotic metabolism, Endocrine Reviews 23 (2002), 687-702.

[5] T. Wada, J. Gao and W. Xie, PXR and CAR in energy metabolism, Trends in Endocrinology \& Metabolism 20 (2009), 273-279

[6] Z. Wei, S. Jiang, Y. Zhang, et al., The effect of microRNAs in the regulation of human CYP3A4: A systematic study using a mathematical model, Scientific Reports 4 (2014), 4283.

[7] Y. Huang, X.J. Shen, Q. Zou, et al., Biological functions of microRNAs: A review, Journal of Physiology and Biochemistry 67 (2011), 129-139.

[8] B.P. Lewis, C.B. Burge and D.P. Bartel, Conserved seed pairing, often flanked by adenosines, indicates that thousands of human genes are microRNA targets, Cell 120 (2005), 15-20.

[9] E. Berezikov, Evolution of microRNA diversity and regulation in animals, Nature Reviews Genetics 12 (2011), 846-860.

[10] A. Ramamoorthy, Y. Liu, S. Philips, et al., Regulation of microRNA expression by rifampin in human hepatocytes, Drug Metabolism and Disposition: The Biological Fate of Chemicals 41 (2013), 1763-1768.

[11] K. Takahashi, N. Tatsumi, T. Fukami, et al., Integrated analysis of rifampicin-induced microRNA and gene expression changes in human hepatocytes, Drug Metabolism and Pharmacokinetics 29 (2014), 333-340. 
[12] T.Y. Miki Nakajima, MicroRNAs from biology to future pharmacotherapy: Regulation of cytochrome P450s and nuclear receptors, Pharmacology \& Therapeutics 131 (2011), 330-337.

[13] Y. Tsuchiya, M. Nakajima, S. Takagi, et al., MicroRNA regulates the expression of human cytochrome P450 1B1, Cancer Research 66 (2006), 9090-9098.

[14] A.L.L. Ramamoorthy, A. Aedigk, L.D. Bradford, E.A. Benson, D.A. Flockhart and T.C. Skaar, In silico and in vitro identification of microRNAs that regulate hepatic nuclear factor 4a expression, Drug Metabolism and Disposition 40 (2012), 726-733.

[15] Y.Z. Pan, W. Gao and A.M. Yu, MicroRNAs regulate CYP3A4 expression via direct and indirect targeting, Drug Metabolism and Disposition: The Biological Fate of Chemicals 37 (2009), 2112-2117.

[16] C.H. Lee, W.H. Kuo, C.C. Lin, et al., MicroRNA-regulated protein-protein interaction networks and their functions in breast cancer, International Journal of Molecular Sciences 14 (2013), 11560-11606.

[17] C.W. Tseng, C.C. Lin, C.N. Chen, et al., Integrative network analysis reveals active microRNAs and their functions in gastric cancer, BMC Systems Biology 5 (2011), 99.

[18] C.C. Lin, J.T. Hsiang, C.Y. Wu, et al., Dynamic functional modules in co-expressed protein interaction networks of dilated cardiomyopathy, BMC Systems Biology 4 (2010), 138.

[19] Y. Benjammini and Y. Hochberg, Controlling the false discovery rate A practical and powerfu, Journal of the Royal Statistical SocietySeries B (Methodological) 57 (1995), 289-300.

[20] R.C. Friedman, K.K. Farh, C.B. Burge, et al., Most mammalian mRNAs are conserved targets of microRNAs, Genome Research 19 (2009), 92-105.

[21]D.M. Garcia, D. Baek, C. Shin, et al., Weak seed-pairing stability and high target-site abundance decrease the proficiency of lsy-6 and other microRNAs, Nature Structural \& Molecular Biology 18 (2011), 1139-1146.

[22] D. Betel, M. Wilson, A. Gabow, D. S. Marks, C. Sander et al., The microRNA.org resource: Targets and expression, Nucleic Acids Research 36 (2008), D149-153.

[23]D.G. Azra Krek, Matthew N. Poy, Rachel Wolf, Lauren Rosenberg, Eric J. Epstein, Philip MacMenamin, Isabelle da Piedade and Kristin C. Gunsalus, Markus stoffel and nikolaus rajewsky, combinatorial microRNA target predictions, Nature Genetics 37 (2005), 495-500

[24] S. Haider, B. Ballester, D. Smedley, et al., BioMart central portal--unified access to biological data, Nucleic Acids Research 37 (2009), W23-27.

[25] T.S. Keshava Prasad, R. Goel, K. Kandasamy, et al., Human protein reference database-2009 update, Nucleic Acids Research 37 (2009), D767-772.

[26] J. Wang, K. Huo, L. Ma, et al., Toward an understanding of the protein interaction network of the human liver, Molecular Systems Biology 7 (2011), 536.

[27] P. Shannon, A. Markiel, O. Ozier, et al., Cytoscape: A software environment for integrated models of biomolecular interaction networks, Genome Research 13 (2003), 2498-2504.

[28] L.A. Lammers, R. Achterbergh, E.M. de Vries, et al., Short-term fasting alters cytochrome p450-mediated drug metabolism in humans, Drug Metabolism and Disposition: The Biological Fate of Chemicals 43 (2015), 819-828.

[29] S.E. Kandel and J.N. Lampe, Role of protein-protein interactions in cytochrome P450-mediated drug metabolism and toxicity, Chemical Research in Toxicology 27 (2014), 1474-1486.

[30] U.M. Zanger, K. Klein, M. Thomas, et al., Genetics, epigenetics, and regulation of drug-metabolizing cytochrome p450 enzymes, Clinical Pharmacology and Therapeutics 95 (2014), 258-261.

[31]M. Ringelhan, R.M. Schmid and F. Geisler, The NF-kappaB subunit RelA/p65 is dispensable for successful liver regeneration after partial hepatectomy in mice, PloS One 7 (2012), e46469.

[32] Y. Du, J. Wang, J. Jia, et al., Human hepatocytes with drug metabolic function induced from fibroblasts by lineage reprogramming, Cell Stem Cell 14 (2014), 394-403.

[33] T. Suzuki, Y. Kanai, T. Hara, et al., Crucial role of the small GTPase ARF6 in hepatic cord formation during liver development, Molecular and Cellular Biology 26 (2006), 6149-6156.

[34]N. Yusa, K. Watanabe, S. Yoshida, et al., Transcription factor Sp3 activates the liver/bone/kidney-type alkaline phosphatase promoter in hematopoietic cells, Journal of Leukocyte Biology 68 (2000), 772-777.

[35] B. Wu, W. Wang, Y. Zhan, et al., CXCL13, CCL4, and sTNFR as circulating inflammatory cytokine markers in primary and SLE-related autoimmune hemolytic anemia, Journal of Translational Medicine 13 (2015), 112.

[36]D.G. Saliba, A. Heger, H.L. Eames, et al., IRF5:RelA interaction targets inflammatory genes in macrophages, Cell Reports 8 (2014), 1308-1317.

[37] S.D. Hsu, Y.T. Tseng, S. Shrestha, et al., MiRTarBase update 2014: An information resource for experimentally validated miRNA-target interactions, Nucleic Acsids Research 42 (2014), D78-85. 